DOI 10.4171/JEMS/355

Yann Bugeaud · Pascal Hubert · Thomas A. Schmidt

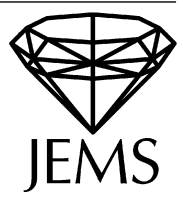

\title{
Transcendence with Rosen continued fractions
}

Received July 13, 2010 and in revised form February 14, 2011

\begin{abstract}
We give the first transcendence results for the Rosen continued fractions. Introduced over half a century ago, these fractions expand real numbers in terms of certain algebraic numbers.
\end{abstract}

Keywords. Rosen continued fractions, Liouville inequality, Hecke groups, transcendence

\section{Introduction}

In 1954, D. Rosen defined an infinite family of continued fraction algorithms [21]. Introduced to aid in the study of certain Fuchsian groups, these continued fractions were applied some thirty years later by J. Lehner [15] in the study of Diophantine approximation by orbits of these groups.

The Rosen continued fractions and variants have been of recent interest. For studies of their dynamical and arithmetical properties, see [8, 19, 12]. For their applications to the study of geodesics on related hyperbolic surfaces, see [24, 7, 18]. For applications to Teichmüller geodesics arising from (Veech) translation surfaces, see [28, 5, 27, 6]. Several basic questions remain open, including that of arithmetically characterizing the real numbers having a finite Rosen continued fraction expansion; see [16, 14, 6]. Background on Rosen continued fractions is given in the next section.

The first transcendence criteria for regular continued fractions were proved by E. Maillet, H. Davenport and K. F. Roth, A. Baker, and recently improved by B. Adamczewski and Y. Bugeaud; see $[1,2,10]$ and the references given there. In particular, Theorem 4.1 of [2] asserts that if $\xi$ is an algebraic irrational number with sequence of convergents $\left(p_{n} / q_{n}\right)_{n \geq 1}$, then the sequence $\left(q_{n}\right)_{n \geq 1}$ cannot increase too rapidly. It is natural to ask whether similar transcendence results can be proven using Rosen continued fractions. We give the first such results.

Y. Bugeaud: Université de Strasbourg, Mathématiques, 7, rue René Descartes, 67084 Strasbourg Cedex, France; e-mail: bugeaud@math.unistra.fr

P. Hubert: LATP, case cour A, Faculté des sciences Saint Jérôme, Avenue Escadrille Normandie Niemen, 13397 Marseille Cedex 20, France; e-mail: hubert@cmi.univ-mrs.fr

T. A. Schmidt: Department of Mathematics, Oregon State University, Corvallis, OR 97331, USA; e-mail: toms@math.orst.edu

Mathematics Subject Classification (2010): 11J70, 11J81 
Theorem 1.1. Fix $\lambda=2 \cos (\pi / m)$ for an integer $m>3$, and denote the field extension degree $[\mathbb{Q}(\lambda): \mathbb{Q}]$ by D. If a real number $\xi \notin \mathbb{Q}(\lambda)$ has an infinite expansion in Rosen continued fraction over $\mathbb{Q}(\lambda)$ of convergents $p_{n} / q_{n}$ satisfying

$$
\limsup _{n \rightarrow \infty} \frac{\log \log q_{n}}{n}>\log (2 D-1)
$$

then $\xi$ is transcendental.

To state our second result, we associate to the Rosen continued fraction expansion

$$
\left[\varepsilon_{1}(x): r_{1}(x), \varepsilon_{2}(x): r_{2}(x), \ldots, \varepsilon_{n}(x): r_{n}(x), \ldots\right]:=\frac{\varepsilon_{1}}{r_{1} \lambda+\frac{\varepsilon_{2}}{r_{2} \lambda+\cdots}}
$$

of a real number $x$ in $[-\lambda / 2, \lambda / 2)$ the sequence of pairs of integers $\left(\varepsilon_{i}, r_{i}\right)_{i \geq 1}$, which we call the partial quotients, and thus consider $\mathcal{A}=\{ \pm 1\} \times \mathbb{N}$ as the alphabet of the Rosen continued fraction expansions.

As usual, we denote the length of a finite word $U=u_{1} \cdots u_{k}$ as $|U|=k$. For any positive integer $s$, we write $U^{s}$ for the word $U \cdots U$ ( $s$-fold concatenation of the word $U$ ). More generally, for any positive real number $s$, we denote by $U^{s}$ the word $U^{\lfloor s\rfloor} U^{\prime}$, where $U^{\prime}$ is the prefix of $U$ of length $\lceil(s-\lfloor s\rfloor)|U|\rceil$.

Just as Adamczewski and Bugeaud [1, 10] showed for regular continued fraction expansions, a real number whose Rosen continued fraction expansion is appropriately "stammering" must be transcendental.

Theorem 1.2. Fix $\lambda=2 \cos (\pi / m)$ for an integer $m>3$, and denote the field extension degree $[\mathbb{Q}(\lambda): \mathbb{Q}]$ by D. Let $\xi$ be an infinite Rosen continued fraction with convergents $\left(p_{n} / q_{n}\right)_{n \geq 1}$ such that

$$
B:=\limsup _{n} q_{n}^{1 / n}<\infty
$$

Write

$$
b:=\liminf _{n} q_{n}^{1 / n} .
$$

Assume that there are two infinite sequences $\left(U_{n}\right)_{n \geq 1}$ and $\left(V_{n}\right)_{n \geq 1}$ of finite words over the alphabet $\mathcal{A}$ and an infinite sequence $\left(w_{n}\right)_{n \geq 1}$ of real numbers greater than 1 such that, for $n \geq 1$, the word $U_{n} V_{n}^{w_{n}}$ is a prefix of the infinite word composed of the partial quotients of $\xi$. If

$$
\limsup _{n \rightarrow \infty} \frac{\left|U_{n}\right|+w_{n}\left|V_{n}\right|}{2\left|U_{n}\right|+\left|V_{n}\right|}>\frac{3 D}{2} \cdot \frac{\log B}{\log b}
$$

then $\xi$ is either (at most) quadratic over $\mathbb{Q}(\lambda)$, or transcendental.

Lemma 2.1 implies that $\log b$ is positive.

The key to our proofs is that both the numerator and denominator of a Rosen convergent dominate their respective conjugates in an appropriate fashion; see Lemma 3.1. From this one can bound the height of a Rosen convergent in terms of its denominator; see Lemma 3.3. Then, exactly as in the case of regular continued fractions, we apply tools 
from Diophantine approximation, namely an extension to number fields of the Roth theorem for the proof of Theorem 1.1, and the Schmidt Subspace Theorem for the proof of Theorem 1.2.

Both theorems are weaker than their analogues for regular continued fractions, since we must work in a number field of degree $D$ rather than in the field $\mathbb{Q}$. However, for $m=4$ and $m=6$, that is, for $\lambda_{4}=\sqrt{2}$ and $\lambda_{6}=\sqrt{3}$, our results can be considerably strengthened and we can get, essentially, the exact analogues of the results established for regular continued fractions. The key point is that, in both cases, for every convergent $p_{n} / q_{n}$, exactly one of $p_{n}, q_{n}$ is in $\mathbb{Z}$, the other being in $\lambda \mathbb{Z}$; see Remark 2 below.

\section{Background}

\subsection{Rosen fractions}

We set $\lambda=\lambda_{m}=2 \cos (\pi / m)$ and $\mathbb{I}_{m}=[-\lambda / 2, \lambda / 2)$ for $m \geq 3$. For a fixed integer $m \geq 3$, the Rosen continued fraction map is defined by

$$
T(x)= \begin{cases}\left|\frac{1}{x}\right|-\lambda\left\lfloor\left|\frac{1}{\lambda x}\right|+\frac{1}{2}\right\rfloor, & x \neq 0, \\ 0, & x=0,\end{cases}
$$

for $x \in \mathbb{I}_{m}$; here and below, we omit the index $m$ whenever it is clear from context. For $n \geq 1$, we define

$$
\varepsilon_{n}(x)=\varepsilon\left(T^{n-1} x\right) \quad \text { and } \quad r_{n}(x)=r\left(T^{n-1} x\right)
$$

with

$$
\left.\varepsilon(y)=\operatorname{sgn}(y) \quad \text { and } \quad r(y)=\left\lfloor\mid \frac{1}{\lambda y}\right\rfloor+\frac{1}{2}\right\rfloor .
$$

Then, as Rosen showed in [21], the Rosen continued fraction expansion of $x$ is given by

$$
\left[\varepsilon_{1}(x): r_{1}(x), \varepsilon_{2}(x): r_{2}(x), \ldots, \varepsilon_{n}(x): r_{n}(x), \ldots\right]:=\frac{\varepsilon_{1}}{r_{1} \lambda+\frac{\varepsilon_{2}}{r_{2} \lambda+\cdots}} .
$$

As usual we define the convergents $p_{n} / q_{n}$ of $x \in \mathbb{I}_{m}$ by

$$
\left(\begin{array}{ll}
p_{-1} & p_{0} \\
q_{-1} & q_{0}
\end{array}\right)=\left(\begin{array}{ll}
1 & 0 \\
0 & 1
\end{array}\right)
$$

and

$$
\left(\begin{array}{cc}
p_{n-1} & p_{n} \\
q_{n-1} & q_{n}
\end{array}\right)=\left(\begin{array}{cc}
0 & \varepsilon_{1} \\
1 & \lambda r_{1}
\end{array}\right)\left(\begin{array}{cc}
0 & \varepsilon_{2} \\
1 & \lambda r_{2}
\end{array}\right) \cdots\left(\begin{array}{cc}
0 & \varepsilon_{n} \\
1 & \lambda r_{n}
\end{array}\right)
$$

for $n \geq 1$. From this definition it is immediate that $\left|p_{n-1} q_{n}-q_{n-1} p_{n}\right|=1$, and that the well-known recurrence relations

$$
\begin{aligned}
& p_{-1}=1, \quad p_{0}=0, \quad p_{n}=\lambda r_{n} p_{n-1}+\varepsilon_{n} p_{n-2}, n \geq 1, \\
& q_{-1}=0, \quad q_{0}=1, \quad q_{n}=\lambda r_{n} q_{n-1}+\varepsilon_{n} q_{n-2}, n \geq 1,
\end{aligned}
$$


hold. It also follows that

$$
\frac{p_{n}}{q_{n}}=\left[\varepsilon_{1}: r_{1}, \varepsilon_{2}: r_{2}, \ldots, \varepsilon_{n}: r_{n}\right]
$$

and

We define

$$
\frac{q_{n-1}}{q_{n}}=\left[1: r_{n}, \varepsilon_{n}: r_{n-1}, \ldots, \varepsilon_{2}: r_{1}\right]
$$

$$
M_{n}=\left(\begin{array}{cc}
p_{n-1} & p_{n} \\
q_{n-1} & q_{n}
\end{array}\right), \quad \text { and find that } \quad x=M_{n} \cdot T^{n}(x),
$$

where $\cdot$ denotes the usual fractional linear operation, namely

$$
x=\frac{p_{n-1} T^{n}(x)+p_{n}}{q_{n-1} T^{n}(x)+q_{n}} .
$$

\subsection{Approximation with Rosen fractions}

We briefly discuss the convergence of the "convergents" to $x$. One can rephrase some of Rosen's original arguments in terms of the (standard number-theoretic) natural extension map $\mathcal{T}(x, y)=\left(T(x), \frac{1}{r \lambda+\varepsilon y}\right)$ where $r=r_{1}(x)$ and $\varepsilon=\varepsilon_{1}(x)$. The "mirror formula" (3) shows that $\mathcal{T}^{n}(x, 0)=\left(T^{n}(x), q_{n-1} / q_{n}\right)$. Extending earlier work of H. Nakada, it is shown in [8] that $\mathcal{T}(x, y)$ has planar domain $\Omega$ with $y$-coordinates between 0 and $R=R(\lambda)$, where $R=1$ if the index $m$ is even, and otherwise $R$ is the positive root of $R^{2}+(2-\lambda) R-1=0$, in which case we have $1>R>\lambda / 2$ (see Lemma 3.3 of [8]). Therefore, the sequence $\left(q_{n}\right)_{n \geq 1}$ is strictly increasing. But, as Rosen mentions, if $x$ has infinite expansion, then either $\varepsilon_{n}=1$ or $r_{n}>1$ occurs infinitely often; from this one finds that $q_{n} \geq 1$ for all $n$ and that the limit of $q_{n}$ as $n \rightarrow \infty$ is infinite.

One easily adapts Rosen's arguments to deduce the following.

Lemma 2.1. For every $x \in \mathbb{I}_{m}$ of infinite expansion, we have

$$
\liminf _{n} q_{n}^{1 / n}>1
$$

Proof. We know that the sequence $\left(q_{n}\right)_{n \geq 1}$ increases and that, if either $\varepsilon_{n}=1$ or $r_{n}>1$, then $q_{n}>\lambda q_{n-1}$. Furthermore, there are no more than $h$ consecutive indices $i$ with $\left(\varepsilon_{i}, r_{i}\right)=(-1,1)$, with $h=m / 2$ or $(m-3) / 2$ depending on the parity of $m$; see [21] or [8]. Consequently, for any $n$, there is some $i=1, \ldots, h+1$ such that $q_{n+i}>\lambda q_{n+i-1}$, giving

$$
q_{n+h+1} \geq q_{n+i}>\lambda q_{n+i-1} \geq \lambda q_{n} .
$$

As $q_{1} \geq \lambda$, letting $s(n)=1+\left\lfloor\frac{n-1}{h+1}\right\rfloor$, we have $q_{n} \geq \lambda^{s(n)}$. Since $\lambda>1$, this proves the lemma.

Remark 2.2. In fact, H. Nakada [20] shows that for almost all such $x, \lim _{n \rightarrow \infty} \frac{1}{n} \log q_{n}$ exists, being equal to half the entropy of $T$. He also shows that the entropy equals $C \cdot(m-2) \pi^{2} /(2 m)$ where $C=1 / \log (1+R)$ when $m$ is odd, and equals $1 / \log [(1+\cos (\pi / m)) / \sin (\pi / m)]$ when $m$ is even. This $C$ is the normalizing constant of the invariant measure with density $(1+x y)^{-2}$ on the domain $\Omega$ of the planar natural extension $\mathcal{T}$; see [8]. 
Rosen also gave bounds on $\left|x-p_{n} / q_{n}\right|$. Using (4), one can get

$$
\left|x-\frac{p_{n}}{q_{n}}\right|=\frac{1}{q_{n}^{2}} \frac{1}{\left|q_{n+1} / q_{n}+T^{n+1} x\right|},
$$

from which Nakada (see [20, Lemma 4]) finds

$$
\frac{1}{q_{n}\left(q_{n+1}+q_{n}\right)} \leq\left|x-\frac{p_{n}}{q_{n}}\right| \leq \frac{c_{1}}{q_{n}^{2}},
$$

with $c_{1}=c_{1}(\lambda)=R /(1-R \lambda / 2)$. (The lower bound is in [21].)

Now, from (4) one also finds

$$
\left|x-\frac{p_{n}}{q_{n}}\right|=\frac{1}{q_{n} q_{n+1}} \frac{1}{\left|1+\frac{q_{n}}{q_{n+1}} T^{n+1} x\right|} .
$$

Since the closed, compact planar region $\Omega$ is of finite measure with respect to the measure with density $(1+x y)^{-2}$, it follows that $\mathcal{T}^{n+1}(x, 0)$ remains a bounded distance from the curve $y=-1 / x$. Thus, there is some $c_{2}$ such that

$$
\left|x-\frac{p_{n}}{q_{n}}\right|<\frac{c_{2}}{q_{n} q_{n+1}} .
$$

Rosen, arguing differently, gave $c_{2}=1 /(1-\lambda / 2)$; in particular, convergence of the approximation sequence follows. Rosen's value is not optimal. To see this, one combines Proposition 4.1 of [8] with the approach of Theorems 4.4 and 4.5 there (depending on parity of $m$ ).

\subsection{Traces in Hecke groups}

Rosen introduced his continued fractions to study Hecke groups. The Hecke (triangle Fuchsian) group $G_{m}$ with $m \in\{3,4,5, \ldots\}$ is the group generated by

$$
\left(\begin{array}{cc}
1 & \lambda_{m} \\
0 & 1
\end{array}\right) \text { and }\left(\begin{array}{cc}
0 & -1 \\
1 & 0
\end{array}\right)
$$

with $\lambda_{m}$ as above. The Rosen expansion of a real number terminates at a finite term if and only if $x$ is a parabolic fixed point of $G_{m}$; see [21]. These points are clearly contained in $\mathbb{Q}\left(\lambda_{m}\right)$ but in general there are elements of this field that have infinite Rosen expansion; see $[16,14,6]$.

Remark 2.3. The values of finite Rosen expansions form the set $G_{m} \cdot \infty$, which is in fact a subset of $\lambda \mathbb{Q}\left(\lambda^{2}\right) \cup\{\infty\}$. To see this, one uses induction on word length in the generators displayed above - an ordered pair $(a, c)$ giving a column of any element of $G_{m}$ must be such that exactly one element of the pair is in $\mathbb{Z}\left[\lambda^{2}\right]$, and the other is in $\lambda \mathbb{Z}\left[\lambda^{2}\right]$. Note that this also applies to convergents $p_{n} / q_{n}$ : exactly one of $p_{n}, q_{n}$ is in $\mathbb{Z}\left[\lambda^{2}\right]$, the other being in $\lambda \mathbb{Z}\left[\lambda^{2}\right]$. 
When $m=3$, we have $G_{3}=\operatorname{PSL}(2, \mathbb{Z})$. In general each $G_{m}$ is isomorphic to the free product of a cyclic group of order two and a cyclic group of order $m$. Recall that a Fuchsian triangle group is generated by even words in the reflections about the sides of some hyperbolic triangle. Thus any Fuchsian triangle group is of index two in the group generated by these reflections; for each $G_{m}$, we denote this larger group by $\Delta_{m}$.

Since $\lambda_{m}$ is the sum of the root of unity $\zeta_{2 m}:=\exp 2 \pi i /(2 m)$ with its complex conjugate, $\mathbb{Q}\left(\lambda_{m}\right)$ is a number field of degree $d:=\phi(2 m) / 2$ over the rationals, where $\phi$ denotes the Euler totient function.

The following key phenomenon property of Hecke groups can be shown in various manners. The result holds for a larger class of groups, from Corollary 5 of [26], due to [11] (extending the arguments from $G_{m}$ to $\Delta_{m}$ is straightforward). Independent of this earlier work, Bogomolny-Schmit [7] gave a clever proof of the result specifically for $\Delta_{m}$. See the next remark for another perspective.

Theorem 2.4. Fix $m$ as above, and let $\Delta_{m}$ be the full reflection group in which $G_{m}$ has index two. Then for any $M \in \Delta_{m}$ whose trace is of absolute value greater than 2 , we have

$$
|\operatorname{tr}(M)| \geq|\sigma(\operatorname{tr}(M))|
$$

where $\sigma$ is any field embedding of $\mathbb{Q}\left(\lambda_{m}\right)$.

Remark 2.5. This result can be proven "geometrically". Up to conjugacy, each of the Hecke groups appears as the Veech group of some translation surface; see [28]. Those elements of trace greater than 2 in absolute value are the "derivatives" of the affine pseudoAnosov diffeomorphisms of the surface. The dilatation of a pseudo-Anosov $\phi$ is the dominant eigenvalue $\lambda$ of the action of $\phi$ on the integral homology of the underlying surface. (The other eigenvalues are hence conjugates of $\lambda$.) The corresponding element of the Veech group has trace of absolute value $\lambda+\lambda^{-1}$ from which it follows that this trace dominates its conjugates.

\subsection{Approximation by algebraic numbers}

The following result was announced by Roth [23] and proven by LeVeque; see Chapter 4 of [17]. (The version below is Theorem 2.5 of [9].) Recall that given an algebraic number $\alpha$, its naive height, denoted by $H(\alpha)$, is the largest absolute value of the coefficients of its minimal polynomial over $\mathbb{Z}$.

Theorem 2.6 (Roth-LeVeque). Let $K$ be a number field, and $\xi$ a real algebraic number not in $K$. Then, for any $\epsilon>0$, there exists a positive constant $c(\xi, K, \epsilon)$ such that

$$
|\xi-\alpha|>\frac{c(\xi, K, \epsilon)}{H(\alpha)^{2+\epsilon}} \quad \text { for every } \alpha \text { in } K
$$

The logarithmic Weil height of $\alpha$ lying in a number field $K$ of degree $D$ over $\mathbb{Q}$ is $h(\alpha)=$ $D^{-1} \sum_{\nu} \log ^{+} \max _{v \in M_{K}}\|\alpha\|_{\nu}$, where $\log ^{+} t$ equals 0 if $t \leq 1$ and $M_{K}$ denotes the places (finite and infinite "primes") of the field, and $\|\cdot\|_{\nu}$ is the $v$-absolute value. This definition 
is independent of the field $K$ containing $\alpha$. Recall that the product formula states that the product over $v \in M_{K}$ of the $\|\alpha\|_{\nu}$ equals 1 . Using this, for $\alpha, \beta \in K$ with $\beta \neq 0$ one has

$$
h(\alpha / \beta) \leq \sum_{\sigma} \frac{1}{D} \log \max \{|\sigma(\alpha)|,|\sigma(\beta)|\},
$$

where the sum is taken over the field embeddings $\sigma$ of $K$ into the complex numbers, and $|\cdot|$ denotes the usual complex norm.

The two heights are related by

$$
\log H(\alpha) \leq \operatorname{deg}(\alpha) h(\alpha)+\log 2
$$

for any non-zero algebraic number $\alpha$; see Lemma 3.11 from [29].

We recall a consequence of the W. Schmidt Subspace Theorem; see Theorem 9A of [25].

Theorem 2.7. Let $d$ be a positive integer and $\xi$ be a real algebraic number of degree greater than $d$. Then, for every positive $\varepsilon$, there exist only finitely many algebraic numbers $\alpha$ of degree at most $d$ such that

$$
|\xi-\alpha|<H(\alpha)^{-d-1-\varepsilon} .
$$

Note that the Roth theorem is exactly the case $d=1$ of Theorem 2.7.

In the proof of Theorem 1.2, we could apply Theorem 2.7, but the algebraic numbers $\alpha$ which we use to approximate $\xi$ are of degree at most 2 over a fixed number field. In this situation, the next theorem, kindly communicated to us by J.-H. Evertse [13], yields a stronger result than the previous one.

Theorem 2.8 (Evertse). Let $K$ be a real algebraic number field of degree $d$. Let $t$ be a positive integer and $\xi$ be a real algebraic number of degree greater than $t$ over $K$. Then, for every positive $\varepsilon$, there exist only finitely many algebraic numbers $\alpha$ of degree tover $K$ and $\delta$ over $\mathbb{Q}$ such that

$$
|\xi-\alpha|<H(\alpha)^{-d t(t+1+\varepsilon) / \delta} .
$$

Note that Theorem 2.8 extends Theorem 2.6.

\subsection{Sturmian sequences: towards an application of Theorem 1.2}

To give an explicit family of Rosen expansions satisfying the hypotheses of Theorem 1.2, we recall a result of [3] on Sturmian sequences.

Let $a$ and $b$ be letters in some alphabet. The complexity function of a sequence $\mathbf{u}=$ $u_{1} u_{2} \ldots$ with values in $\{a, b\}$ is given by letting $p(n, \mathbf{u})$ be the number of distinct words of length $n$ that occur in $\mathbf{u}$. A sequence $\mathbf{u}$ is called Sturmian if its complexity satisfies $p(n, \mathbf{u})=n+1$ for all $n$. As Arnoux [4] writes, one can obtain any such sequence by taking a ray with irrational slope in the real plane and intersecting it with an integral grid, assigning $a$ when the ray intersects a horizontal grid line and $b$ when it meets a vertical grid line. Indeed, the slope of a Sturmian sequence is the density of $a$ in the sequence (one shows that the limit as $n$ tends to infinity of the average of the number of occurrences $a$ in $u_{1} \cdots u_{n}$ exists; see [4, Proposition 6.1.10]). 
Lemma 2.9. Let $\mathbf{u}$ be a Sturmian sequence whose slope has an unbounded regular continued fraction expansion. Then, for every positive integer $n$, there are finite words $U, V$ and a positive real number s such that $U V^{s}$ is a prefix of $\mathbf{u}$ and $\left|U V^{s}\right| \geq n|U V|$.

Proof. This follows from the proof of Proposition 11.1 from [3].

Remark 2.10. We apply the above lemma to Sturmian sequences where both $a, b$ are of the form $(\varepsilon, r)$, with $\varepsilon= \pm 1$ and $r \in \mathbb{N}$. In particular, we use this in the context of Rosen expansions to prove Corollary 4.1 .

\section{Bounding the height of convergents}

In what follows, we fix $\lambda=\lambda_{m}$ for some $m>3$, and suppose that $\xi \in(0, \lambda / 2)$ is a real algebraic number having an infinite Rosen continued fraction expansion over $\mathbb{Q}(\lambda)$. Our goal is to estimate the naive height $H\left(p_{n} / q_{n}\right)$ of the $n$th convergent $p_{n} / q_{n}$. In light of Theorem 2.4, we let $n_{0}$ be the least value of $n$ such that $q_{n}>2$.

Lemma 3.1. Let $c_{3}=c_{3}(\lambda)$ be defined by $c_{3}=\min _{\sigma}|\sigma(\lambda)| / \lambda$, where the minimum is taken over all field embeddings of $\mathbb{Q}(\lambda)$ into $\mathbb{R}$. Then for all $n \geq n_{0}$, and any such $\sigma$,

$$
q_{n} \geq c_{3}\left|\sigma\left(q_{n}\right)\right| \text { and } p_{n} \geq c_{3}\left|\sigma\left(p_{n}\right)\right| \text {. }
$$

Proof. For any $n \geq n_{0}$, recall that $M_{n}=\left(\begin{array}{ll}p_{n-1} & p_{n} \\ q_{n-1} & q_{n}\end{array}\right)$; this is clearly an element of $\Delta_{m}$.

By Theorem 2.4 we have $q_{n}+p_{n-1} \geq\left|\sigma\left(q_{n}+p_{n-1}\right)\right|$.

Now let $j \in \mathbb{N}$ and set

$$
M_{n, j}=\left(\begin{array}{ll}
p_{n-1} & p_{n} \\
q_{n-1} & q_{n}
\end{array}\right)\left(\begin{array}{cc}
1 & j \lambda \\
0 & 1
\end{array}\right)=\left(\begin{array}{ll}
p_{n-1} & p_{n}+j \lambda p_{n-1} \\
q_{n-1} & q_{n}+j \lambda q_{n-1}
\end{array}\right) .
$$

This is also an element of $\Delta_{m}$ of trace greater than 2 , and hence

$$
\left|p_{n-1}+q_{n}+j \lambda q_{n-1}\right| \geq\left|\sigma\left(p_{n-1}+q_{n}\right)+j \sigma\left(\lambda q_{n-1}\right)\right| .
$$

Since this holds for all positive $j$, we must have $\lambda q_{n-1} \geq\left|\sigma\left(\lambda q_{n-1}\right)\right|$. That is,

$$
q_{n-1} \geq \frac{|\sigma(\lambda)|}{\lambda}\left|\sigma\left(q_{n-1}\right)\right| \geq\left(\min _{\sigma} \frac{|\sigma(\lambda)|}{\lambda}\right)\left|\sigma\left(q_{n-1}\right)\right| .
$$

Similarly, using

$$
N_{n, j}=\left(\begin{array}{ll}
p_{n-1} & p_{n} \\
q_{n-1} & q_{n}
\end{array}\right)\left(\begin{array}{cc}
1 & 0 \\
j \lambda & 1
\end{array}\right)=\left(\begin{array}{ll}
p_{n-1}+j \lambda p_{n} & p_{n} \\
q_{n-1}+j \lambda q_{n} & q_{n}
\end{array}\right),
$$

we find

$$
p_{n} \geq \frac{|\sigma(\lambda)|}{\lambda}\left|\sigma\left(p_{n}\right)\right| \geq\left(\min _{\sigma} \frac{|\sigma(\lambda)|}{\lambda}\right)\left|\sigma\left(p_{n}\right)\right| .
$$


Remark 3.2. We conjecture that in fact $q_{n}$ is always greater than or equal to its conjugates, thus in the above one can replace $c_{3}$ by 1 .

Lemma 3.3. Let $D$ denote the field extension degree $[\mathbb{Q}(\lambda): \mathbb{Q}]$. There exists a constant $c_{4}=c_{4}(\lambda)$ such that for all $n \geq n_{0}$,

$$
H\left(p_{n} / q_{n}\right) \leq c_{4} q_{n}^{D}
$$

Proof. Since $p_{n}$ and $q_{n}$ are algebraic integers of degree at most $D$, it follows from Lemma 3.1 and (6) that

$$
h\left(p_{n} / q_{n}\right) \leq \sum_{\sigma} \frac{1}{D} \log \max \left\{\left|\sigma\left(p_{n}\right)\right|,\left|\sigma\left(q_{n}\right)\right|\right\}
$$

where $\sigma$ runs through the complex embeddings. We thus have

$$
h\left(p_{n} / q_{n}\right) \leq c_{4}^{\prime}+\log q_{n}
$$

for a suitable positive constant $c_{4}^{\prime}$. Using (7), we get the asserted estimate.

Lemma 3.4. Let $\alpha$ be a real number in $[-\lambda / 2, \lambda / 2)$ with an ultimately periodic expansion in a Rosen continued fraction. Denote by $\left(p_{n} / q_{n}\right)_{n \geq 1}$ the sequence of its convergents. Denote by $\mu$ the length of the preperiod and by $v$ the length of the period, with the convention that $\mu=0$ if the expansion is purely periodic. Then $\alpha$ is of degree at most 2 over $\mathbb{Q}(\lambda)$, and there exists $c_{5}=c_{5}(\lambda, \alpha)$ such that

$$
H(\alpha) \leq c_{5}\left(q_{\mu} q_{\mu+\nu}\right)^{D} .
$$

Proof. In the notation of (4), $\alpha$ is fixed by $M=M_{\mu}^{-1} M_{\mu+\nu}$. It thus satisfies a quadratic equation with entries in $\mathbb{Z}[\lambda]$, and hence is of degree at most 2 over $\mathbb{Q}(\lambda)$. Indeed, $\alpha$ is a root of $f(x)=c x^{2}+(d-a) x-b$ with $a, b, c, d$ denoting the entries of $M$. Each entry is a $\mathbb{Z}$-linear combination of monomials of the form $r s$ with $r$ an entry of $M_{\mu}$ and $s$ an entry of $M_{\mu+v}$.

Now, $\alpha$ is also a root of $\tilde{f}(x)=\prod_{\sigma} \sigma(f)(x) \in \mathbb{Z}[x]$, where $\sigma(f)$ denotes the result of applying $\sigma$ to the coefficients of $f(x)$. By Lemma 3.1, all of the conjugates of each of $p_{\mu}, p_{\mu-1}, q_{\mu-1}, q_{\mu}$ can be bounded by the product of $q_{\mu}$ with a constant depending upon $\alpha$ and $\lambda$. Similarly for the entries of $M_{\mu+v}$. After some computation, we conclude that the height of $\alpha$ is $\ll q_{\mu}^{D} q_{\mu+v}^{D}$. (One checks that the case of $\mu=0$ is subsumed by the above.)

Remark 3.5. Whereas a real number whose regular continued fraction expansion is ultimately periodic is exactly of degree two over the field of rational numbers, in the previous lemma the words "at most" are necessary. Indeed, $x=1$ has an ultimately periodic Rosen expansion with respect to any $\lambda_{m}$ with $m$ even; see [21]. Further examples of elements of $\mathbb{Q}\left(\lambda_{m}\right)$ with periodic expansions are easily given when $m \in\{4,6\}$; see Corollary 1 of [24]. Further examples, including cases with $m \in\{7,9\}$, are given in [22, 14]. 


\section{Transcendence results}

As usual, « and $\gg$ denote inequalities with an implied constant.

\subsection{Applying Roth-LeVeque: the proof of Theorem 1.1}

We now show that the sequence of denominators of convergents to an algebraic number cannot grow too quickly. Theorem 1.1 then follows.

Let $\varepsilon$ be a positive real number. Let $\zeta$ be an algebraic number having an infinite Rosen expansion with convergents $r_{n} / s_{n}$.

By the Roth-LeVeque Theorem 2.6, we have

$$
\left|\zeta-r_{n} / s_{n}\right| \gg H\left(r_{n} / s_{n}\right)^{-2-\varepsilon} \quad \text { for } n \geq 1 .
$$

Hence by Lemma 3.3, for $n \geq n_{0}=n_{0}(\zeta)$, we have $\left|\zeta-r_{n} / s_{n}\right| \gg s_{n}^{-2 D-D \varepsilon}$. Inequality (5) then gives that there exists a constant $c_{6}$ (independent of $n \geq n_{0}$ ) such that

$$
s_{n+1}<c_{6} s_{n}^{2 D-1+D \varepsilon} .
$$

Set $a=2 D-1+D \varepsilon$. For $j<n_{0}$, choose $\ell_{j}$ such that $s_{j}<\ell_{j} s_{j-1}^{a}$. We set $c_{7}=$ $\max \left\{1, c_{6}, \ell_{1}, \ldots, \ell_{n_{0}-1}\right\}$ and find that for any $n>1$,

$$
s_{n+1}<c_{7} s_{n}^{a}<c_{7}\left(c_{7} s_{n-1}^{a}\right)^{a} \leq\left(c_{7} s_{n-1}\right)^{a^{2}}
$$

continuing in this manner, we have $s_{n+1}<\left(c_{7} s_{1}\right)^{a^{n}}$. Since $s_{n+1}>s_{n}$, letting $c_{8}=c_{7} s_{1}$ gives $\log s_{n}<a^{n} \log c_{8}$. From this it follows that

$$
\limsup _{n \rightarrow \infty} \frac{\log \log s_{n}}{n}<\log (D(2+\varepsilon)-1) .
$$

Letting $\varepsilon$ go to zero, we see that every algebraic number satisfies

$$
\limsup _{n \rightarrow \infty} \frac{\log \log s_{n}}{n} \leq \log (2 D-1),
$$

as asserted.

\subsection{Proof of Theorem 1.2 and an application}

With $\lambda=2 \cos (\pi / m)$ fixed, given $\xi$ with infinite Rosen continued fraction with convergents $\left(p_{n} / q_{n}\right)_{n \geq 1}$, we let $b=\liminf _{n} q_{n}^{1 / n}$ and $B=\limsup q_{n} q_{n}^{1 / n}$, and assume that $B<\infty$. Let $\eta$ be a positive real number with $b-1<\eta<b$. Since there are only finitely many $n$ with either $q_{n}^{1 / n}<b-\eta$ or $q_{n}^{1 / n}>B+\eta$, we have both $q_{n} \gg(b-\eta)^{n}$ and $q_{n} \ll(B+\eta)^{n}$.

Suppose that $w$ is a positive real number and $U, V$ are finite words in $\{ \pm 1\} \times \mathbb{N}$ such that $U V^{w}$ is a prefix of the infinite word composed of the partial quotients of $\xi$. Denote by $\alpha$ the real number of degree at most two over $\mathbb{Q}(\lambda)$ whose Rosen continued fraction is 
given by the word $U V^{\infty}$, where $V^{\infty}$ means the concatenation of infinitely many copies of $V$. Set $|U|=u$ and $|V|=v$. Since $\xi$ and $\alpha$ have their first $\lfloor u+v w\rfloor$ partial quotients in common, we have

$$
|\xi-\alpha|<c_{1} q_{\lfloor u+v w\rfloor}^{-2} \ll(b-\eta)^{-2(u+v w)} .
$$

Furthermore, it follows from Lemma 3.4 that

$$
H(\alpha) \ll\left(q_{u} q_{u+v}\right)^{D} \ll(B+\eta)^{D(2 u+v)} .
$$

Combined with the previous inequality, this gives

$$
|\xi-\alpha| \ll H(\alpha)^{-2(u+v w) \log (b-\eta) /(D(2 u+v) \log (B+\eta))} .
$$

Now suppose that $\xi$ is algebraic of degree greater than two over $\mathbb{Q}(\lambda)$. Then, for every $\varepsilon>0$, there exists a positive constant $C(\varepsilon)$ such that every real algebraic number $\beta$ of degree at most 2 over $\mathbb{Q}(\lambda)$ satisfies

$$
|\xi-\beta|>C(\varepsilon) H(\beta)^{-3-\varepsilon}
$$

This follows from Theorem 2.6 if $\beta$ is in $\mathbb{Q}(\lambda)$, and otherwise by applying Theorem 2.8 with $t=2$ and $d t=\delta$ to each subfield $K$ of $\mathbb{Q}(\lambda)$.

This proves that $\xi$ must be transcendental if there are $u, v, w$ such that $u+v w$ is arbitrarily large and

$$
\frac{2(u+v w) \log b}{D(2 u+v) \log B}>3
$$

as asserted.

Corollary 4.1. A Rosen continued fraction in $\lambda$ whose sequence of partial quotients is Sturmian with slope of unbounded regular continued fraction partial quotients represents a number that is transcendental or at most quadratic over $\mathbb{Q}(\lambda)$.

Proof. Combine Lemma 2.9 with Theorem 1.2.

Remark 4.2. Using the Subspace Theorem as in [1, 10] does not yield in general an improvement of Theorem 1.2. In case $u=0, b=B$, inequality (1) reduces to $w>3 D / 2$, while, proceeding as in $[1,10]$, we would get $w>2 D-1$. However, if $b$ is much smaller than $B$, and $D$ is small, then the approach of $[1,10]$ presumably gives a slightly better result than Theorem 1.2.

Acknowledgments. We thank both Kariane Calta and the referee for their comments.

The second named author is partially supported by project blanc ANR: ANR-06-BLAN-0038. The third author thanks FRUMAN, Marseille and the Université P. Cézanne. 


\section{References}

[1] Adamczewski, B., Bugeaud, Y.: On the complexity of algebraic numbers II. Continued fractions. Acta Math. 195, 1-20 (2005) Zbl 1195.11093 MR 2233683

[2] Adamczewski, B., Bugeaud, Y.: On the Maillet-Baker continued fractions. J. Reine Angew. Math. 606, 105-121 (2007) Zbl 1145.11054 MR 2337643

[3] Adamczewski, B., Bugeaud, Y.: Nombres réels de complexité sous-linéaire : mesures d'irrationalité et de transcendance. J. Reine Angew. Math. 658, 65-98 (2011) Zbl pre05962773 MR 2831513

[4] Arnoux, P.: Sturmian sequences. In: Substitutions in Dynamics, Arithmetics and Combinatorics, Lecture Notes in Math. 1794, Springer, Berlin, 143-198 (2002) MR 1970391

[5] Arnoux, P., Hubert, P.: Fractions continues sur les surfaces de Veech. J. Anal. Math. 81, 35-64 (2000) Zbl 1029.11035 MR 1785277

[6] Arnoux, P., Schmidt, T. A.: Veech surfaces with non-periodic directions in the trace field. J. Modern Dynam. 3, 611-629 (2009) Zbl 1186.37050 MR 2587089

[7] Bogomolny, E., Schmit, C.: Multiplicities of periodic orbit lengths for non-arithmetic models. J. Phys. A 37, 4501-4526 (2004) Zbl 1050.37008 MR 2065941

[8] Burton, R., Kraaikamp, C., Schmidt, T. A.: Natural extensions for the Rosen fractions. Trans. Amer. Math. Soc. 352, 1277-1298 (1999) Zbl 0938.11036 MR 1650073

[9] Bugeaud, Y.: Approximation by Algebraic Numbers. Cambridge Tracts in Math. 160, Cambridge Univ. Press, Cambridge (2004) Zbl 1055.11002 MR 2136100

[10] Bugeaud, Y.: Automatic continued fractions are transcendental or quadratic. Ann. Sci. École Norm. Sup., to appear

[11] Cohen, P., Wolfart, J.: Modular embeddings for some nonarithmetic Fuchsian groups. Acta Arith. 56, 93-110 (1990) Zbl 0717.14014 MR 1075639

[12] Dajani, K., Kraaikamp, C., Steiner, W.: Metrical theory for $\alpha$-Rosen fractions. J. Eur. Math. Soc. 11, 1259-1283 (2009) Zbl 1184.28016 MR 2557135

[13] Evertse, J.-H.: personal communication

[14] Hanson, E., Merberg, A., Towse, C., Yudovina, E.: Generalized continued fractions and orbits under the action of Hecke triangle groups. Acta Arith. 134, 337-348 (2008) Zbl 1219.11105 MR 2449157

[15] Lehner, J.: Diophantine approximation on Hecke groups. Glasgow Math. J. 27, 117-127 (1985) Zbl 0576.10023 MR 0819833

[16] Leutbecher, A.: Über die Heckeschen Gruppen G( $\lambda$ ). Abh. Math. Sem. Hamburg 31, 199-205 (1967) Zbl 0161.27601 MR 0228438

[17] LeVeque, W. J.: Topics in Number Theory, Vols. 1 and 2. Addison-Wesley, Reading, MA (1956) Zbl 1009.11001 MR 1942365

[18] Mayer, D., Strömberg, F.: Symbolic dynamics for the geodesic flow on Hecke surfaces. J. Modern Dynam. 2, 581-627 (2008) Zbl 1161.37031 MR 2449139

[19] Nakada, H.: Continued fractions, geodesic flows and Ford circles. In: Algorithms, Fractals and Dynamics, Y. Takahashi (ed.), Plenum, 179-191 (1995) Zbl 0868.30005 MR 1402490

[20] Nakada, H.: On the Lenstra constant associated to the Rosen continued fractions. J. Eur. Math. Soc. 12, 55-70 (2010) Zbl 1205.11091 MR 2578603

[21] Rosen, D.: A class of continued fractions associated with certain properly discontinuous groups. Duke Math. J. 21, 549-563 (1954) Zbl 0056.30703 MR 0065632

[22] Rosen, D., Towse, C.: Continued fraction representations of units associated with certain Hecke groups. Arch. Math. (Basel) 77, 294-302 (2001) Zbl 0992.11034 MR 1853543

[23] Roth, K. F.: Rational approximations to algebraic numbers. Mathematika 2, 1-20 (1955); corrigendum, 168 Zbl 0064.28501 MR 0072182 
[24] Schmidt, T. A., Sheingorn, M.: Length spectra of the Hecke triangle groups. Math. Z. 220, 369-397 (1995) Zbl 0840.11019 MR 1362251

[25] Schmidt, W. M.: Diophantine Approximation. Lecture Notes in Math. 785, Springer, Berlin (1980) Zbl 0421.10019 MR 0568710

[26] Schmutz Schaller, P., Wolfart, J.: Semi-arithmetic Fuchsian groups and modular embeddings. J. London Math. Soc. (2) 61, 13-24 (2000) Zbl 0968.11022 MR 1408860

[27] Smillie, J., Ulcigrai, C.: Geodesic flow on the Teichmüller disk of the regular octagon, cutting sequences and octagon continued fractions maps. In: Dynamical Numbers-Interplay between Dynamical Systems and Number Theory, Contemp. Math. 532, Amer. Math. Soc., 29-65 (2010) Zbl 1222.37012 MR 2762132

[28] Veech, W. A.: Teichmüller curves in modular space, Eisenstein series, and an application to triangular billiards. Invent. Math. 97, 553-583 (1989) Zbl 0676.32006 MR 1005006

[29] Waldschmidt, M.: Diophantine Approximation on Linear Algebraic Groups. Transcendence properties of the exponential function in several variables. Grundlehren Math. Wiss. 326, Springer, Berlin (2000) Zbl 0944.11024 MR 1756786 